\title{
Trans-interaction of nephrin and Neph1/Neph3 induces cell adhesion that associates with decreased tyrosine phosphorylation of nephrin
}

\author{
Eija Heikkilä*, Mervi Ristola*, Marika Havana ${ }^{\dagger}$, Nina Jones ${ }^{\ddagger}$, \\ Harry Holthöfer ${ }^{\dagger, \S}$ and Sanna Lehtonen* \\ * Department of Pathology and ${ }^{\dagger}$ Bacteriology and Immunology, Haartman Institute, \\ University of Helsinki, Haartmaninkatu 3, 00290 Helsinki, Finland, ${ }^{*}$ Department of \\ Molecular and Cellular Biology, University of Guelph, 50 Stone Road East, Guelph, Ontario, \\ N1G 2W1, Canada. ${ }^{\S}$ Dublin City University, Dublin 9, Ireland.
}

\begin{abstract}
Running title: Nephrin, Neph1 and Neph3 in cell adhesion
Abbreviations used: SD (slit diaphragm), EGFP (Enhanced Green Fluorescent Protein) and CD2AP (CD2-associated protein)

Address correspondence to: Sanna Lehtonen, University of Helsinki, Haartman Institute, Haartmaninkatu 3, 00290 Helsinki, Finland.

Tel:358-9-191 26792 Fax: 358-9-19126675; E-mail: sanna.h.lehtonen@helsinki.fi
\end{abstract}




\section{SYNOPSIS}

Slit diaphragms are specialized junctions between glomerular epithelial cells (podocytes) that are crucial for glomerular ultrafiltration. Ig superfamily members nephrin and Neph1 are essential components of the slit diaphragm, whereas the role of Neph1 homologue Neph3 in the slit diaphragm is unknown. Here we show that Neph3 homodimerizes and heterodimerizes with nephrin and Neph1. We further investigated whether these interactions play a role in cell adhesion by using mouse $\mathrm{L}$ fibroblasts that lack endogenous cell adhesion activity, and found that Neph1 and Neph3 are able to induce cell adhesion alone whereas nephrin needs to trans-interact with Neph1 or Neph3 in order to promote formation of cell-cell contacts. Tyrosine phosphorylation of nephrin was down-regulated after nephrin trans-interacted with either Neph1 or Neph3 leading to formation of cell-cell contacts. We further found that the expression of Neph3 was increased in nephrin deficient mouse podocytes. Our findings show that nephrin and Neph1 or Neph3 trans-interactions promote cell contact formation suggesting that they may also function together in slit diaphragm assembly.

Keywords: podocyte, slit diaphragm, nephrin, Neph family, cell-cell contacts 


\section{INTRODUCTION}

Glomerular podocytes are highly differentiated epithelial cells that play a key role in the regulation of the kidney ultrafiltration function. They form specialized cell-cell contacts, slit diaphragms (SD), which are important for establishment of a sizeselective filter barrier in the kidney. Congenital nephrotic syndrome of the Finnish type is caused by mutations in NPHS1, the gene encoding the SD protein nephrin. Lack of nephrin results in loss of SDs and massive proteinuria beginning before birth [1]. Furthermore, nephrin expression is decreased in acquired human kidney diseases [2-4]. This highlights the importance of nephrin and consequently the SD in regulating the kidney ultrafiltration function.

Neph1, a protein homologous to nephrin, is also essential for the formation of the SD [5]. Nephrin and Neph1 bind to each other via their extracellular domains and this interaction has been suggested to be important for the formation of the SD [6-8]. Another member of Neph family, Neph3, localizes also in the SD, binds to nephrin [9] and is down-regulated in human acquired proteinuric kidney diseases indicating that it may have a role in the maintenance of the SD $[10,11]$. These data suggest that nephrin, Neph1 and Neph3 may form a complex which could be important for SD assembly.

Ig superfamily proteins have been shown to play a role in the formation of cell-cell contacts via homophilic and heterophilic interactions [12,13]. Furthermore, nephrin and Neph1-3 orthologues in Drosophila and C. elegans play a role in junction formation required for complex differentiation processes including muscle (Drosophila myoblast fusion) [14], eye (Drosophila ommatidia formation) [15] and synapse (C. elegans synaptic specificity) [16] development. During these developmental processes they are expressed in different cell types and their heterophilic trans-interactions induce selective cell adhesion which is crucial for the localization and formation of the junctions. Taken together, these findings on nephrin, Neph1 and Neph3 orthologues suggest that their mammalian counterparts might also play a role in cell adhesion. In the present study, we test this hypothesis by investigating the interactions and adhesive properties of nephrin, Neph1 and Neph3.

\section{EXPERIMENTAL}

\section{Antibodies}

Rabbit antibodies against the extracellular domain (\#033) and intracellular domain of nephrin (\#1034) have been described previously in [17] and [18], respectively. Rabbit antibodies against Neph1 and Neph3 (\#1201) have been described in [19] and [10], respectively, and nephrin phospho-specific antibodies pY1193 and pY1217 in [20]. The following commercial primary antibodies were used: rabbit anti-podocin (SigmaAldrich, Saint Louis, MO), rabbit anti-Neph3 (Alpha Diagnostic International, San Antonio, TX), mouse anti-myc (Clontech Laboratories, Mountain View, CA), rabbit anti-GFP (Invitrogen, Carlsbad, CA), mouse anti-phosphotyrosine (Sigma-Aldrich), mouse anti-HA (Covance, Emeryville, CA), mouse anti-actin (Sigma-Aldrich) and mouse anti- $\gamma$-tubulin (Sigma-Aldrich). 


\section{Construction of vectors}

Full-length rat nephrin (NM_022628) with the stop codon and full-length mouse Neph3 (AK049284) without the stop codon were cloned into pcDNA3.1/myc-His (Invitrogen). The extracellular domain of human Neph3 (NP_954649) without the signal peptide covering amino acids (aa) 21-510 was cloned into signal plgplus vector (R\&D Systems, Minneapolis, MN), which contains the CD33 signal sequence and the FC domain of human IgG1 for secreted expression in mammalian cells. The intracellular parts of human nephrin (AAC39687, aa 1087-1241), Neph1 (NP_060710, aa 521-758) and Neph3 (NP_954649, aa 534-708) were cloned into a derivative of pCDM8 vector. The vector contains the leader sequence of CD5, the $\mathrm{CH} 2$ and $\mathrm{CH} 3$ domains of human IgG1 and the transmembrane region of CD7 (sIg7) [21]. Full-length rat nephrin was cloned into pMSCVneo (Clontech Laboratories) retroviral vector. Enhanced Green Fluorescent Protein (EGFP)-tagged full-length Neph3 and EGFP alone were cloned into pMSCVpuro retroviral vector (Clontech Laboratories) and HA-tagged full-length mouse Neph1 (AY243095) into retroviral pBabe-hygro vector [22].

\section{Cell culture}

293T cells (ATCC, Rockville, MD; \# CLR-11268) and L fibroblasts (L-cells, ATCC; \#CCL-1) were maintained in $5 \% \mathrm{CO}_{2}$ at $37^{\circ} \mathrm{C}$ in Dulbecco's Modified Eagle's Medium (glucose $4500 \mathrm{mg} / \mathrm{l}$ ) (Sigma-Aldrich) supplemented with 10\% fetal bovine serum (FBS) (Sigma-Aldrich), $100 \mathrm{U} / \mathrm{ml}$ penicillin and $0.1 \mathrm{mg} / \mathrm{ml}$ streptomycin and 2 $\mathrm{mM}$ glutamine. L-cells were additionally supplemented with $25 \mathrm{mM}$ HEPES, $\mathrm{pH}$ 7.5.

\section{Generation of stable L-cell lines}

Retroviruses were produced by co-transfecting the retroviral constructs Neph3-EGFPpMSCVpuro (L-Neph3-EGFP), EGFP-pMSCVpuro (L-EGFP) or nephrinpMSCVneo (L-nephrin) and pKAT [23] into 293T cells using Lipofectamine 2000 (Invitrogen). Alternatively, Phoenix Ampho packaging cells (from Garry Nolan, Stanford University, Stanford, CA) were transfected with retroviral constructs Neph1HA-pBabe-hygro (L-Neph1-HA) or pBabe-hygro (L-Mock). After 48 h supernatants were harvested, filtered through $0.45 \mu \mathrm{m}$ nitrocellulose filters (Millipore, Billerica, MA) and supplemented with $10 \mu \mathrm{g} / \mathrm{ml}$ polybrene (Sigma-Aldrich). The supernatants were added to L-cells plated on previous day and centrifuged $1360 \mathrm{~g}$ at RT for 30 min. Medium was changed back to normal after $16 \mathrm{~h}$ and antibiotic selection with puromycin $(10 \mu \mathrm{g} / \mathrm{ml}), \mathrm{G} 418(1 \mathrm{mg} / \mathrm{ml})$ or hygromycin $(0.5 \mathrm{mg} / \mathrm{ml})$ was started $24 \mathrm{~h}$ after infection. Puromycin concentration was gradually increased up to $60 \mu \mathrm{g} / \mathrm{ml}$ and hygromycin up to $1 \mathrm{mg} / \mathrm{ml}$ during two weeks. To establish stable cell lines expressing nephrin and Neph1 or Neph3 in combination, L-cells stably expressing nephrin were infected with retroyiruses carrying Neph3-EGFP or Neph1-HA as described above.

\section{Nephrin TRAP mice}

Nephrin TRAP mice (nephrin ${ }^{\text {trap/trap }}$ ) have been described previously [24]. All experiments were performed according to the European Communities Council Directive 86/609/EEC, and the experiments had approval of the Experimental Animal Committee of the University of Helsinki and the Provincial Government of Southern Finland. 


\section{Preparation of L-cell and E17 kidney lysates}

Cells were lysed on ice in RIPA-lysis buffer ( $150 \mathrm{~mm} \mathrm{NaCl}, 1 \% \mathrm{NP}-40,0.5 \%$ sodium deoxycholate, $0.1 \%$ sodium dodecyl sulfate (SDS), $50 \mathrm{mM}$ Tris- $\mathrm{HCl}, \mathrm{pH} 8.0$ ) supplemented with Complete EDTA-free proteinase inhibitor cocktail (Roche, Mannheim, Germany). The lysates were centrifuged and total protein concentrations were measured by using Bradford assay (Bio-Rad Laboratories, Hercules, CA) according to manufacturer's instructions. Lysates were boiled in Laemmli sample buffer and immunoblotted.

Mouse kidneys from nephrin ${ }^{\text {trap/trap }}$ and nephrin ${ }^{\text {wt/wt }}$ embryos at E17 were lysed on ice using a glass homogenizer in RIPA-lysis buffer supplemented with Complete EDTAfree proteinase inhibitor cocktail (Roche). Insoluble material was removed by centrifugation, and total protein concentrations were measured followed by immunoblotting of the samples.

\section{Transfections and co-immunoprecipitations}

$293 \mathrm{~T}$ cells were transiently transfected with indicated plasmid DNAs using Lipofectamine 2000. $48 \mathrm{~h}$ after transfection, cells were washed with PBS and lysed on ice in $150 \mathrm{mM} \mathrm{NaCl}, 20 \mathrm{mM}$ Tris-HCl pH 7.5, $1 \%$ Nonidet P-40 (NP-40) supplemented with Complete EDTA-free proteinase inhibitor cocktail (Roche), 50 $\mathrm{mM}$ sodium fluoride and $1 \mathrm{mM}$ sodium orthovanadate (NP-40 lysis buffer). After pelleting insoluble material by centrifugation at $15800 \mathrm{~g}$ for $15 \mathrm{~min}$, total protein concentrations were measured. For immunoprecipitations with full-length constructs, cell lysates were first pre-cleared with protein G- or A-sepharose (Zymed Laboratories, South San Francisco, CA) at $4^{\circ} \mathrm{C}$ for $1 \mathrm{~h}$ followed by overnight incubation with anti-myc and anti-nephrin antibodies, or purified mouse or rabbit IgG (Zymed Laboratories). The lysates were then incubated with protein G- or Asepharose at $4^{\circ} \mathrm{C}$ for $1 \mathrm{~h}$. In immunoprecipitations with membrane-bound intracellular fusion proteins, preclearing step was omitted and cell lysates containing equal amounts of protein were incubated with protein G-sepharose at $4^{\circ} \mathrm{C}$ for overnight. Finally, the beads were washed four times with lysis buffer, boiled in Laemmli sample buffer and immunoblotted with anti-myc or anti-nephrin (\#1034) antibodies.

\section{Pull-down assays}

Signal plgplus vector containing the extracellular domain of Neph3 was transfected into 293T cells using Lipofectamine 2000. After $48 \mathrm{~h}$ the Neph3 fusion protein was purified from the cell culture medium by using protein A-sepharose. Glomeruli were isolated from rat kidneys by graded sieving as described [25]. Briefly, the minced rat kidney cortices were passed through sieves with pore sizes of $250 \mu \mathrm{m}, 150 \mu \mathrm{m}$ and 75 $\mu \mathrm{m}$ and glomeruli obtained were pelleted by centrifugation ( $\geq 95 \%$ purity). For pulldown assays, rat glomeruli were lysed on ice with glass homogenizer in NP-40 lysis buffer. Insoluble material was removed and total protein concentrations were measured. Glomerular lysate was pre-cleared with protein A-sepharose at $4^{\circ} \mathrm{C}$ for $1 \mathrm{~h}$ followed by $3 \mathrm{~h}$ incubation with $20 \mu \mathrm{g}$ of fusion proteins of the extracellular domain of Neph3 or Ig-control conjugated with protein A-sepharose. The beads were washed four times with lysis buffer, boiled in Laemmli sample buffer and immunoblotted with anti-nephrin (\#1034) and anti-podocin antibodies. 


\section{Hanging drop assay}

L-cells were grown for $24 \mathrm{~h}$, trypsinized and 15000 cells in $30 \mu \mathrm{l}$ drops of media were placed onto lids of culture dishes filled with PBS to prevent drying of the drops. After $24 \mathrm{~h}\left(\mathrm{~N}_{24}\right)$, the hanging drop cultures were gently pipetted up and down 10 times or they were trypsinized $\left(\mathrm{N}_{\mathrm{T}}\right)$ to count the total number of cells. Cells were then fixed with $2.5 \%$ glutaraldehyde and photographed with phase contrast microscope. For quantification, a particle was defined as more than four cells in a cell aggregate. The total particle number was counted at each time point and the aggregation index was calculated according to the formula $\left(\mathrm{N}_{\mathrm{T}}-\mathrm{N}_{24}\right) / \mathrm{N}_{\mathrm{T}}$ [26]. Two-tailed paired $t$-test was used for statistical analysis.

To measure nephrin tyrosine phosphorylation from cell aggregates, after $24 \mathrm{~h}$, the hanging drops were collected on ice and centrifuged at $320 \mathrm{~g}$ at $4{ }^{\circ} \mathrm{C}$ for $5 \mathrm{~min}$, lysed on ice in NP-40 lysis buffer followed by removal of insoluble material and measurement of total protein concentrations as described above. To measure the total tyrosine phosphorylation of nephrin, immunoprecipitation was performed from cell lysates with anti-nephrin antibody (\#1034) followed by immunoblotting with antinephrin and anti-phosphotyrosine antibodies. To investigate the phosphorylation status of Tyr1204 and Tyr1228 sites in rat nephrin, cell lysates from hanging drop cultures were immunoblotted with anti-nephrin antibody (\#1034) and nephrin phospho-specific antibodies pY1193 (human Y1193=rat Y1204) and pY1217 (human Y1217=rat Y1228).

\section{Immunoblotting}

Proteins were resolved in $10 \%$ or $7.5 \%$ SDS-polyacrylamide gels, transferred to PVDF (Millipore) and blocked with 5\% skimmed milk in PBS or with Odyssey blocking buffer (LI-COR Biosciences, Bad Homburg, Germany) at RT for $1 \mathrm{~h}$. Membranes were incubated at $4^{\circ} \mathrm{C}$ overnight or at RT for $1 \mathrm{~h}$ with the primary antibodies diluted in $1 \%$ skimmed milk in PBS or in Odyssey blocking buffer supplemented with $0.1 \%$ Tween-20. Membranes were washed with $0.2 \%$ Tween-20 in PBS followed by $1 \mathrm{~h}$ incubation at RT with Alexa Fluor 680 (Invitrogen) or IRDye 680 or 800 (LI-COR Biosciences) anti-mouse or anti-rabbit secondary antibodies diluted in $1 \%$ skimmed milk in PBS or in Odyssey blocking buffer supplemented with $0.1 \%$ Tween-20 and $0.01 \%$ SDS. After repeated washes with $0.2 \%$ Tween-20 in PBS, the signal was detected using the Odyssey infrared imaging system (LI-COR Biosciences) and quantification was performed using Odyssey software.

\section{Immunofluorescence microscopy}

L-cells cultured on glass coverslips were fixed with acetone for $10 \mathrm{~min}$ at $-20^{\circ} \mathrm{C}$ or with 4\% PFA for 15 min at RT for HA-staining of Neph1-HA. Hanging drops were fixed with acetone for 20 min at RT. Samples were washed with PBS and blocked in the blocking solution (2\% FBS, 2\% BSA, $0.2 \%$ fish gelatine in PBS) at RT. Cells were incubated with anti-nephrin (\#1034) or anti-HA (Covance) antibodies diluted in the blocking solution for $1 \mathrm{~h}$ at RT followed by washes in PBS and incubation with TRITC-conjugated goat anti-rabbit IgG and TRITC-conjugated donkey anti-mouse IgG (Jackson Laboratories). Specimens were mounted in Mowiol and viewed with Olympus AX70 microscope (Olympus, Tokyo, Japan).

For surface staining of nephrin, L-nephrin cells on glass coverslips were incubated with an antibody directed against the extracellular domain of nephrin (\#033) diluted in 
5\% FBS in PBS on ice for 15 min. Cells were then washed with PBS for 3 min with several changes followed by fixing with 4\% PFA at RT for 10 min. After washes in PBS, samples were blocked with 5\% FBS in PBS for $15 \mathrm{~min}$ at RT and incubated with TRITC-conjugated goat anti-rabbit IgG (Jackson Laboratories) for $15 \mathrm{~min}$ at RT. After washes in PBS, samples were mounted in Mowiol and viewed with Leica TCS SP2 AOBS confocal microscope (Leica Microsystems GmbH, Wetzlar, Germany).

Dissected kidneys from nephrin ${ }^{\text {trap/trap }}$ and nephrin ${ }^{\text {wt/wt }}$ embryos at E17 were immediately snap frozen in liquid nitrogen. Cryosections $(6 \mu \mathrm{m})$ were fized with icecold acetone for $2 \mathrm{~min}$ and blocked with Cas Block (Zymed Laboratories) for $10 \mathrm{~min}$ followed by overnight incubation at $4{ }^{\circ} \mathrm{C}$ with rabbit anti-nephrin (\#1034) and antiNeph3 (Alpha Diagnostic International) antibodies diluted in ChemMate antibody diluent (DakoCytomation, Glostrup, Denmark). Primary antibodies were visualized with TRITC-conjugated goat anti-rabbit IgG and Olympus AX70 microscope.

\section{RESULTS}

\section{Neph3 homodimerizes and heterodimerizes with nephrin and Neph1}

Neph3 localizes to SDs and shares high homology with Neph1 [10,11]. Neph1 interacts with nephrin [6,7], which led us to investigate by reciprocal coimmunoprecipitation assay whether Neph3 also binds to nephrin. We overexpressed nephrin and myc-tagged Neph3 in 293T cells and performed immunoprecipitation from the cell lysates with nephrin and myc antibodies. We found that Neph3-myc coprecipitated with nephrin and reciprocally, nephrin was found in Neph3-myc precipitates (Figure 1A). Control experiments with rabbit and mouse IgG were negative (Figure 1A).

To determine whether the extracellular domains of nephrin and Neph3 mediate their interaction, pull-down assays on glomerular lysates were performed by using a fusion protein containing the extracellular domain of Neph3. The extracellular domain of Neph3 was able to pull down nephrin and podocin from rat glomerular lysate, whereas the vector control was negative (Figure 1B). To further analyze whether Neph3 binds to nephrin also via the intracellular domain, immunoprecipitation assays were performed from 293T cells which were co-transfected with plasmids containing full-length Neph3-myc and the intracellular domain of nephrin. The results showed that the intracellular domain of nephrin precipitated Neph3-myc (Figure 1C). Similarly, the intracellular domains of Neph3 and Neph1 were able to precipitate fulllength Neph3-myc (Figure 1C). The control precipitation containing the intracellular domain of PKD1 was negative (Figure 1C). Collectively, the results indicate that since Neph3 is able to bind to the intracellular domains of nephrin and Neph1 as well as to the extracellular domain of nephrin, Neph3 may be part of nephrin-Neph1 protein complex.

\section{The expression and localization of nephrin, Neph1 and Neph3 in L-cells}

Mouse $L$ fibroblasts (L-cells) do not have endogenous cell adhesion activity due to lack of expression of adherens junction proteins. Therefore they have been widely used to evaluate the adhesive properties of proteins including several members of the Ig and cadherin superfamilies [27,28]. To investigate if nephrin, Neph1 or Neph3 can promote cell-cell contact formation, we established L-cells stably expressing nephrin, 
HA-tagged Neph1 and EGFP-tagged Neph3 by retroviral infection and antibiotic selection. To investigate how co-expression of nephrin together with either Neph1 or Neph3 in the same cell affects cell adhesion, we infected L-nephrin cells with retroviruses containing Neph1-HA (L-nephrin-Neph1-HA) or Neph3-EGFP (Lnephrin-Neph3-EGFP). The expression of the proteins in L-cells was investigated by immunoblot analysis which showed that nephrin, EGFP and Neph3-EGFP (Figure 2A) as well as Neph1-HA (Figure 2B) migrated at their expected molecular weights, and that all proteins were expressed at equal levels in both singly and doubly transfected cells. Immunofluorescence microscopy revealed that nephrin was mainly localized on free membrane edges (Figure 2C, arrows) and its localization on the plasma membrane was confirmed by surface staining using an antibody directed against the extracellular domain of nephrin (Figure 2D). Neph3-EGFP (Figure 2E, arrows) and Neph1-HA (Figure 2F, arrow) were found at cell-cell contacts and EGFP was localized in cytoplasm (Figure $2 \mathrm{G}$ ).

Neph1 and Neph3 show both homophilic adhesion activity and heterophilic adhesion activity with nephrin

To investigate whether nephrin and Neph1 can promote cell adhesion, the adhesive properties of L-nephrin, L-Neph1-HA or control L-cells (L-Mock) were examined by hanging drop assay. Neph1-HA expressing L-cells showed 2.1-fold $(\mathrm{p}<0.001)$ increase in aggregation index compared to L-Mock cells, whereas L-nephrin cells showed similar aggregation index as L-Mock cells (Figure 3A). Similarly, coexpression of nephrin and Neph1 (L-nephrin-Neph1-HA) in the same cell did not increase cell adhesion compared to L-Mock cells (Figure 3A). To further investigate whether trans-interaction of nephrin and Neph1 induces cell adhesion, we used Lnephrin and L-Neph1-HA cells in combination in hanging drop assay and found 3fold $(\mathrm{p}<0.001)$ increase in aggregation index compared to L-Mock cells (Figure 3A).

To investigate whether Neph3 induces cell adhesion alone or in cooperation with nephrin, we performed similar assays as described above using L-nephrin, L-Neph3EGFP, L-nephrin-Neph3-EGFP and L-EGFP cells. Similar to Neph1, Neph3 alone showed 2.3-fold $(\mathrm{p}<0.001)$ increase and after trans-interacting with nephrin, 3-fold $(\mathrm{p}<0.001)$ increase in cell aggregation compared to control cells (L-EGFP) (Figure 3B). Simultaneous expression of Neph3 and nephrin in L-cells (L-nephrin-Neph3EGFP) showed a minor 1.4 -fold increase $(\mathrm{p}<0.01)$ in aggregation index compared to control cells (Figure 3B).

To confirm that the aggregated cells in hanging drops contained both L-nephrin and L-Neph3-EGFP cells, immunofluorescence stainings were performed on cell aggregates. Both Neph3-EGFP and nephrin were found at cell junctions where they co-localized (Figure 3C). Nephrin staining was punctate whereas Neph3-EGFP appeared as a linear sharp line at cell-cell contacts. The results indicate that Neph1 and Neph3 are homophilic adhesion molecules and furthermore, their transinteractions with nephrin further induce their adhesion activity.

\section{Trans-interaction between nephrin and Neph1 or Neph3 leads to a decrease in} tyrosine phosphorylation of nephrin

Tyrosine phosphorylation of nephrin has been shown to induce different cellular processes including actin polymerization [29,30], elevation of intracellular calcium levels [31] and raft-mediated endocytosis of nephrin [32]. This suggests that cell 
adhesion might also be associated with changes in nephrin tyrosine phosphorylation. We therefore investigated whether cell adhesion induced by the interaction of nephrin with Neph1 or Neph3 affects the tyrosine phosphorylation status of nephrin. To this end, we immunoprecipitated nephrin from equal amount of hanging drop culture lysates and immunoblotted the precipitates with anti-nephrin (\#1034) and antiphosphotyrosine antibodies. Relative tyrosine phosphorylation of nephrin was quantified by normalizing the amount of phosphotyrosine to total amount of nephrin in nephrin immunoprecipitates. Our results showed that the total tyrosine phosphorylation of nephrin was not significantly different between L-nephrin, Lnephrin-Neph3-EGFP and L-nephrin-Neph1-HA cells in hanging drop cultures (Figure 4A). However, hanging drop cultures containing mixed cell populations of Lnephrin and L-Neph3-EGFP showed 51\% $(\mathrm{p}<0.01)$ decrease in the total tyrosine phosphorylation of nephrin compared to L-nephrin cells. Similarly, hanging drop cultures containing L-nephrin and L-Neph1-HA showed 48\% ( $\mathrm{p}<0.05)$ decrease in the total tyrosine phosphorylation of nephrin compared to L-nephrin cells (Figure 4A). LEGFP cells were negative for nephrin and phosphotyrosine in immunoblotting. Similar decrease in the tyrosine phosphorylation of nephrin was observed when using another nephrin antibody (\#033) to immunoprecipitate nephrin (data not shown).

The cytoplasmic region of rat nephrin contains seven tyrosine phosphorylation sites of which Tyr1204 (Tyr1193 in human and Tyr1208 in mouse) and Tyr1228 (Tyr1217 in human and Tyr1232 in mouse) are flanked by conserved amino acid motifs which, upon phosphorylation of tyrosine residues, are able to bind to $\mathrm{SH} 2$ domain containing cytosolic proteins including Nck1/2 and PLC- $\gamma$ which induce actin polymerization and $\mathrm{Ca}^{2+}$ signalling, respectively [29-31] (Figure 4B). To investigate whether the decrease in tyrosine phosphorylation of nephrin is due to de-phosphorylation of Tyr1204 and Tyr1228, quantitative immunoblot analysis was performed from cell lysates of hanging drop cultures using nephrin and phospho-specific nephrin antibodies pY1193 and pY1217 [20], which recognize phosphorylated Tyr1204 and Tyr1228 sites of rat nephrin, respectively. Immunoblotting of lysates prepared from nephrin expressing L-cell hanging drop cultures with pY1193 and pY1217 antibodies showed a positive signal whereas immunoblots of L-Mock and L-EGFP were negative confirming the specificity of the antibodies (Figures 4C-D). Quantitative immunoblot analysis normalizing the amount of tyrosine-phosphorylated nephrin to total amount of nephrin showed that nephrin phosphorylation at Tyr1204 and Tyr1228 sites stayed at similar levels in combination of L-nephrin and L-Neph1-HA cells and in combination of L-nephrin and L-Neph3-EGFP cells compared to L-nephrin cells in hanging drop cultures (Figures 4E-F). These observations suggest that the interactions between nephrin and Neph1 or Neph3 inducing the formation of cell-cell contacts result in reduced tyrosine phosphorylation of nephrin and that the decrease in nephrin phosphorylation occurs at sites other than Tyr1204 and Tyr1228.

\section{The expression of Neph3 is increased in nephrin ${ }^{\text {trap/trap }}$ mouse kidneys}

Even though nephrin deficient mice cannot form SDs, the podocyte foot processes of these mice are interconnected by narrow junctions [24]. Since we found that Neph1 and Neph3 can form cell-cell contacts alone in hanging drop assays in L-cells, we investigated the expression of Neph1 and Neph3 in nephrin deficient and control mouse kidneys by quantitative immunoblot analysis. The results showed that Neph3 expression was increased 4-fold $(\mathrm{p}<0.01)$ in nephrin deficient mouse kidneys (Figures 5A-B). Neph1 also showed 2-fold up-regulation, but the increase was not statistically 
significant $(\mathrm{p}<0.14)$. Podocin protein levels were similar in nephrin deficient and control mouse kidneys and nephrin expression was practically absent in nephrin deficient mouse kidneys as expected (Figures 5A-B). We also investigated the localization of Neph3 by immunfluorescence microscopy and found that Neph3 localizes in the podocytes lining the capillary loops in both wildtype (nephrin ${ }^{+/+}$) and nephrin deficient kidneys (nephrin ${ }^{\text {trap/trap }}$ ) (Figure 5C). Nephrin localized in podocytes in wildtype kidneys and was not detected in nephrin deficient mouse kidneys as expected (Figure 5C).

\section{DISCUSSION}

It has been proposed that the interactions between nephrin and Neph family members may be important for the formation of the unique cell-cell contact, the SD. In the present study we investigated the role of nephrin, Neph1 and Neph3 in cell adhesion both individually and in different combinations. We demonstrated that nephrin acts cooperatively with Neph1 and/or Neph3 in cell adhesion via trans-interactions, and that this cell adhesion activity is accompanied by a decrease in tyrosine phosphorylation of nephrin. We also found that Neph1 and Neph3 can form cell-cell contacts in a homophilic fashion whereas nephrin alone cannot. Our results also showed that Neph3 was up-regulated in nephrin deficient mouse kidneys.

The extracellular domains of nephrin and Neph1 interact with each other and this interaction has been suggested to induce adhesion between opposing podocytes and thus lead to the formation of the SD $[6,8]$. We found that Neph3 was also able to bind to the extracellular domain of nephrin suggesting that it may contribute to the formation of the SD together with nephrin. Here we show that the extracellular interactions between nephrin and Neph1/Neph3 play a role in cell contact formation as cell adhesion was induced when nephrin on the cell surface interacted with Neph1 or Neph3 on the surface of another cell. In addition to extracellular domain-mediated interactions, nephrin binds to Neph1 via its intracellular domain [7]. Similarly, we found that Neph3 was able to bind to the intracellular domain of nephrin and also to that of Neph1. Moreover, Neph3 was able to homodimerize via its intracellular domain. These results indicate that Neph1 and Neph3 can cis-heterodimerize with nephrin on the plasma membrane of the same cell. However, in hanging drop aggregation assay, when we co-expressed nephrin together with Neph1 or Neph3 in the same cell, cell aggregation was not affected or was induced only slightly indicating that heterophilic cis-interactions between nephrin and Neph1 or Neph3 do not play a remarkable role in cell adhesion. Aggregation assay, however, revealed that in addition to heterophilic trans-interactions of Neph1 or Neph3 with nephrin, homophilic trans-interactions of Neph1 or Neph3 with themselves also induced cell aggregation. The heterophilic trans-interactions induced stronger cell aggregation than the homophilic trans-interactions. These data indicate that heterophilic transinteractions between nephrin and Neph family members may be more important for cell adhesion than their homophilic trans-interactions or heterophilic cis-interactions. In a similar fashion, heterophilic trans-interactions of the nectin family of $\mathrm{Ig}$ superfamily proteins promote cell adhesion more efficiently than their homophilic trans-interactions [28]. Interestingly, Garg et al. have shown that cis-heterodimers of nephrin and Neph1 play a role in another cellular process, namely inducing reorganization of the actin cytoskeleton, by showing that clustering of nephrin together with Neph1 on the plane of the plasma membrane induces actin polymerization [33]. 
Collectively, these data suggest that dynamic organization of nephrin and Nephfamily proteins in different protein complexes on the plasma membrane induce distinct cellular pathways.

The extracellular domain of nephrin has been shown to homodimerize by biochemical assays $[6,7,34]$ and Khoshnoodi et al. found that nephrin promotes cell-cell adhesion in HEK293 cells [34]. By contrast, in Jurkat cells [9], as well as in our model of mouse L fibroblasts, nephrin was not able to induce cell adhesion. Khoshnoodi et al. analyzed nephrin adhesion in adherent cells growing on culture plates whereas we used a quantitable hanging drop cell aggregation assay. L-cells cannot normally form cell-cell contacts due to lack of adherens junction proteins, whereas HEK293 cells are adherent cells which express junctional proteins and aggregate in hanging drop cultures even without nephrin (our unpublished data). Collectively, the data suggest that nephrin alone can not induce cell-cell aggregation in adhesion-deficient cells but can promote cell adhesion in adherent cells expressing proteins contributing to cell contact formation. In terms of heterophilic trans-interactions, Nishida et al. proposed that trans-interaction between nephrin and Neph3 does not induce cell adhesion in Jurkat cells [9], whereas we found that nephrin cooperates with Neph3 in cell adhesion in trans-configuration using L-cells. These variations may be due to differences in cell types and/or techniques used.

In addition to inducing signalling cascades which lead to actin polymerization $[29,30]$, nephrin participates in pathways that lead to elevation of cytosolic calcium levels [31] and raft-mediated endocytosis of nephrin [32]. In all the above studies, signalling was initiated by clustering of nephrin on the plane of the plasma membrane which results in tyrosine phosphorylation of nephrin by Src kinase Fyn. This tyrosine phosphorylation is needed for nephrin to interact with $\mathrm{SH} 2$ domain containing adapter proteins Nck1/2 and PLC- $\gamma$ which results in actin polymerization $[29,30]$ and calcium signalling [31], respectively. SH2 domain binding sites for Nck1/2 and PLC- $\gamma$ are conserved on human, mouse and rat nephrin (Tyr1204 and Tyr1228 in rat, Figure 4B) and mouse and human nephrin contain an additional Nck1/2 SH2 domain binding site (human Tyr1176 = mouse Tyr1191). Our results with rat nephrin show that when nephrin forms cell-cell contacts with Neph1 or Neph3 in trans-configuration, the total tyrosine phosphorylation of nephrin decreases. However, the phosphorylation level of the conserved SH2 signalling sites Tyr1204 and Tyr1228 in rat nephrin was not decreased after nephrin formed cell-cell contacts with Neph3 or Neph1.

The intracellular domain of rat nephrin contains seven tyrosine phosphorylation sites suggesting that the overall reduced tyrosine phosphorylation of nephrin observed when nephrin trans-interacts with Neph1 or Neph3 leading to cell-cell contact formation is due to dephosphorylation of tyrosine residues other than Tyr1204 and Tyr1228. Of the five additional tyrosine residues, phosphorylation of Tyr1152 has been shown to induce binding of phosphoinositide 3-kinase regulatory p85 subunit and activate Akt pathway [35] but the function of the other four phosphorylation sites in rat nephrin is unknown. Further studies are needed to define which tyrosine residues on nephrin are dephosphorylated upon nephrin and Neph1 or nephrin and Neph3 mediated cell-cell contact formation. Since the previously described SH2 domain mediated signalling cascades do not appear to be involved in nephrin and Neph1 or nephrin and Neph3 dependent cell adhesion, it will be of interest to identify 
the pathways involved in regulating nephrin- and Neph-family- mediated cell adhesion activities.

Lack of nephrin in mice leads to loss of SDs [24,36], but surprisingly, the expression of essential SD proteins including CD2-associated protein (CD2AP) and podocin is preserved in nephrin deficient podocytes [37]. Here we found, however, that Neph3 was up-regulated in nephrin deficient mouse kidneys. The result indicates that even though Neph3 structurally resembles nephrin and is up-regulated when nephrin is absent, it cannot compensate for the function of nephrin in the formation of the SD. Nephrin deficient podocytes are interconnected by narrow junctions [24] and further, Done et al. showed that tight-junction protein claudin-3 is up-regulated in nephrin deficient podocyte junctions [37], suggesting that these podocyte cell contacts share characteristics of tight junctions. Since we found that Neph3 is able to induce cell aggregation and thereby function in cell-cell contact formation, the up-regulation of Neph3 in nephrin deficient podocytes may indicate that without nephrin, Neph3 could participate together with other podocyte molecules in the formation of the junctional complexes between podocyte foot processes.

Collectively, the present study shows that Neph3 is part of the nephrin-Neph1 protein complex and provides novel insights of how nephrin, Neph1 and Neph3 function in cell adhesion either alone or in cooperation. Further investigations are necessary to understand the relevance of the cell adhesion activities of nephrin, Neph1 and Neph3 for the formation of the SD in vivo and the detailed molecular mechanisms involved.

\section{ACKNOWLEDGEMENTS}

Prof Gerd Walz and Prof Peter Gerke are acknowledged for providing Neph1 antibody and mouse Neph1 cDNA. Ulla Kiiski is acknowledged for excellent technical assistance with embryonic kidney tissues and immunofluorescence stainings of the hanging drops.

\section{FUNDING}

This study was supported by grants from Academy of Finland (114963, 121248 and 218021; SL), Biomedicum Helsinki Foundation (EH), Finnish Cultural Foundation (EH, MR), Finnish Kidney Foundation (EH, MR), Helsinki Biomedical Graduate School (MR), Jenny and Antti Wihuri Foundation (EH), Maud Kuistila Memorial Foundation (EH), Orion Pharma Foundation (EH) and Paulo Foundation (EH). NJ is the recipient of a Kidney Research Scientist Core Education and National Training (KRESCENT) Program New Investigator Award and a Biomedical Research Grant from the Kidney Foundation of Canada. 


\section{REFERENCES}

1 Kestila, M., Lenkkeri, U., Mannikko, M., Lamerdin, J., McCready, P., Putaala, H., Ruotsalainen, V., Morita, T., Nissinen, M., Herva, R., Kashtan, C. E., Peltonen, L., Holmberg, C., Olsen, A. and Tryggvason, K. (1998) Positionally cloned gene for a novel glomerular protein--nephrin--is mutated in congenital nephrotic syndrome. Mol. Cell. 1, 575-582

2 Doublier, S., Ruotsalainen, V., Salvidio, G., Lupia, E., Biancone, L., Conaldi, P. G., Reponen, P., Tryggvason, K. and Camussi, G. (2001) Nephrin redistribution on podocytes is a potential mechanism for proteinuria in patients with primary acquired nephrotic syndrome. Am. J. Pathol. 158, 1723-1731

3 Koop, K., Eikmans, M., Baelde, H. J., Kawachi, H., De Heer, E., Paul, L. C. and Bruijn, J. A. (2003) Expression of podocyte-associated molecules in acquired human kidney diseases. J. Am. Soc. Nephrol. 14, 2063-2071

4 Wang, S. X., Rastaldi, M. P., Patari, A., Ahola, H., Heikkila, E. and Holthofer, H. (2002) Patterns of nephrin and a new proteinuria-associated protein expression in human renal diseases. Kidney Int. 61, 141-147

5 Donoviel, D. B., Freed, D. D., Vogel, H., Potter, D. G., Hawkins, E., Barrish, J. P., Mathur, B. N., Turner, C. A., Geske, R., Montgomery, C. A., Starbuck, M., Brandt, M., Gupta, A., Ramirez-Solis, R., Zambrowicz, B. P. and Powell, D. R. (2001) Proteinuria and perinatal lethality in mice lacking NEPH1, a novel protein with homology to NEPHRIN. Mol. Cell. Biol. 21, 4829-4836

6 Gerke, P., Huber, T. B., Sellin, L., Benzing, T. and Walz, G. (2003) Homodimerization and heterodimerization of the glomerular podocyte proteins nephrin and NEPH1. J. Am. Soc. Nephrol. 14, 918-926

7 Barletta, G. M., Kovari, I. A., Verma, R. K., Kerjaschki, D. and Holzman, L. B. (2003) Nephrin and Neph1 co-localize at the podocyte foot process intercellular junction and form cis hetero-oligomers. J. Biol. Chem. 278, 19266-19271

8 Liu, G., Kaw, B., Kurfis, J., Rahmanuddin, S., Kanwar, Y. S. and Chugh, S. S. (2003) Neph1 and nephrin interaction in the slit diaphragm is an important determinant of glomerular permeability. J. Clin. Invest. 112, 209-221

9 Nishida, K., Hoshino, M., Kawaguchi, Y. and Murakami, F. (2010) Ptf1a directly controls expression of immunoglobulin superfamily molecules nephrin and Neph3 in the developing central nervous system. J. Biol. Chem. 285, 373-380

10 Ihalmo, P., Palmen, T., Ahola, H., Valtonen, E. and Holthofer, H. (2003) Filtrin is a novel member of nephrin-like proteins. Biochem. Biophys. Res. Commun. 300, 364-370

11 Ihalmo, P., Schmid, H., Rastaldi, M. P., Mattinzoli, D., Langham, R. G., Luimula, P., Kilpikari, R., Lassila, M., Gilbert, R. E., Kerjaschki, D., Kretzler, M. and Holthofer, H. (2007) Expression of filtrin in human glomerular diseases. Nephrol. Dial. Transplant. 22, 1903-1909

12 Obrink, B. (1997) CEA adhesion molecules: Multifunctional proteins with signalregulatory properties. Curr. Opin. Cell Biol. 9, 616-626

13 Irie, K., Shimizu, K., Sakisaka, T., Ikeda, W. and Takai, Y. (2004) Roles and modes of action of nectins in cell-cell adhesion. Semin. Cell Dev. Biol. 15, 643656

14 Dworak, H. A., Charles, M. A., Pellerano, L. B. and Sink, H. (2001) Characterization of drosophila hibris, a gene related to human nephrin. Development. 128, 4265-4276 
15 Bao, S. and Cagan, R. (2005) Preferential adhesion mediated by hibris and roughest regulates morphogenesis and patterning in the drosophila eye. Dev. Cell. 8, 925-935

16 Shen, K., Fetter, R. D. and Bargmann, C. I. (2004) Synaptic specificity is generated by the synaptic guidepost protein SYG-2 and its receptor, SYG-1. Cell. 116, 869-881

17 Holthofer, H., Ahola, H., Solin, M. L., Wang, S., Palmen, T., Luimula, P., Miettinen, A. and Kerjaschki, D. (1999) Nephrin localizes at the podocyte filtration slit area and is characteristically spliced in the human kidney. Am. J. Pathol. 155, 1681-1687

18 Ahola, H., Heikkila, E., Astrom, E., Inagaki, M., Izawa, I., Pavenstadt, H., Kerjaschki, D. and Holthofer, H. (2003) A novel protein, densin, expressed by glomerular podocytes. J. Am. Soc. Nephrol. 14, 1731-1737

19 Sellin, L., Huber, T. B., Gerke, P., Quack, I., Pavenstadt, H. and Walz, G. (2003) NEPH1 defines a novel family of podocin interacting proteins. FASEB J. 17, 115-117

20 Jones, N., New, L. A., Fortino, M. A., Eremina, V., Ruston, J., Blasutig, I. M., Aoudjit, L., Zou, Y., Liu, X., Yu, G. L., Takano, T., Quaggin, S. E. and Pawson, T. (2009) Nck proteins maintain the adult glomerular filtration barrier. J. Am. Soc. Nephrol. 20, 1533-43

21 Tsiokas, L., Kim, E., Arnould, T., Sukhatme, V. P. and Walz, G. (1997) Homoand heterodimeric interactions between the gene products of PKD1 and PKD2. Proc. Natl. Acad. Sci. U. S. A. 94, 6965-6970

22 Morgenstern, J. P. and Land, H. (1990) Advanced mammalian gene transfer: High titre retroviral vectors with multiple drug selection markers and a complementary helper-free packaging cell line. Nucleic Acids Res. 18, 35873596

23 Finer, M. H., Dull, T. J., Qin, L., Farson, D. and Roberts, M. R. (1994) Kat: A high-efficiency retroviral transduction system for primary human $\mathrm{T}$ lymphocytes. Blood. 83, 43-50

24 Rantanen, M., Palmen, T., Patari, A., Ahola, H., Lehtonen, S., Astrom, E., Floss, T., Vauti, F., Wurst, W., Ruiz, P., Kerjaschki, D. and Holthofer, H. (2002) Nephrin TRAP mice lack slit diaphragms and show fibrotic glomeruli and cystic tubular lesions. J. Am. Soc. Nephrol. 13, 1586-1594

25 Orlando, R. A., Takeda, T., Zak, B., Schmieder, S., Benoit, V. M., McQuistan, T., Furthmayr, H. and Farquhar, M. G. (2001) The glomerular epithelial cell antiadhesin podocalyxin associates with the actin cytoskeleton through interactions with ezrin. J. Am. Soc. Nephrol. 12, 1589-1598

26 Thoreson, M. A., Anastasiadis, P. Z., Daniel, J. M., Ireton, R. C., Wheelock, M. J., Johnson, K. R., Hummingbird, D. K. and Reynolds, A. B. (2000) Selective uncoupling of p120(ctn) from E-cadherin disrupts strong adhesion. J. Cell Biol. 148, 189-202

27 Nagafuchi, A., Shirayoshi, Y., Okazaki, K., Yasuda, K. and Takeichi, M. (1987) Transformation of cell adhesion properties by exogenously introduced E-cadherin cDNA. Nature. 329, 341-343

28 Satoh-Horikawa, K., Nakanishi, H., Takahashi, K., Miyahara, M., Nishimura, M., Tachibana, K., Mizoguchi, A. and Takai, Y. (2000) Nectin-3, a new member of immunoglobulin-like cell adhesion molecules that shows homophilic and heterophilic cell-cell adhesion activities. J. Biol. Chem. 275, 10291-10299 
29 Verma, R., Kovari, I., Soofi, A., Nihalani, D., Patrie, K. and Holzman, L. B. (2006) Nephrin ectodomain engagement results in src kinase activation, nephrin phosphorylation, nck recruitment, and actin polymerization. J. Clin. Invest. 116, 1346-1359

30 Jones, N., Blasutig, I. M., Eremina, V., Ruston, J. M., Bladt, F., Li, H., Huang, H., Larose, L., Li, S. S., Takano, T., Quaggin, S. E. and Pawson, T. (2006) Nck adaptor proteins link nephrin to the actin cytoskeleton of kidney podocytes. Nature. 440, 818-823

31 Harita, Y., Kurihara, H., Kosako, H., Tezuka, T., Sekine, T., Igarashi, T., Ohsawa, I., Ohta, S. and Hattori, S. (2009) Phosphorylation of nephrin triggers $\mathrm{Ca} 2+$ signaling by recruitment and activation of phospholipase C-\{gamma $\} 1 . \mathrm{J}$. Biol. Chem. 284, 8951-8962

32 Qin, X. S., Tsukaguchi, H., Shono, A., Yamamoto, A., Kurihara, H. and Doi, T. (2009) Phosphorylation of nephrin triggers its internalization by raft-mediated endocytosis. J. Am. Soc. Nephrol. 20, 2534-2545

33 Garg, P., Verma, R., Nihalani, D., Johnstone, D. B. and Holzman, L. B. (2007) Neph1 cooperates with nephrin to transduce a signal that induces actin polymerization. Mol. Cell. Biol. 27, 8698-8712

34 Khoshnoodi, J., Sigmundsson, K., Ofverstedt, L. G., Skoglund, U., Obrink, B., Wartiovaara, J. and Tryggvason, K. (2003) Nephrin promotes cell-cell adhesion through homophilic interactions. Am. J. Pathol. 163, 2337-2346

35 Zhu, J., Sun, N., Aoudjit, L., Li, H., Kawachi, H., Lemay, S. and Takano, T. (2008) Nephrin mediates actin reorganization via phosphoinositide 3-kinase in podocytes. Kidney Int. 73, 556-566

36 Putaala, H., Soininen, R., Kilpelainen, P., Wartiovaara, J. and Tryggvason, K. (2001) The murine nephrin gene is specifically expressed in kidney, brain and pancreas: Inactivation of the gene leads to massive proteinuria and neonatal death. Hum. Mol. Genet. 10, 1-8

37 Done, S. C., Takemoto, M., He, L., Sun, Y., Hultenby, K., Betsholtz, C. and Tryggvason, K. (2008) Nephrin is involved in podocyte maturation but not survival during glomerular development. Kidney Int. 73, 697-704 


\section{FIGURE LEGENDS}

Figure 1. Neph3 homodimerizes and heterodimerizes with nephrin and Neph1. (A) Full-length nephrin and Neph3-myc were co-transfected into 293T cells and nephrin and Neph3 were immunoprecipitated with anti-nephrin and anti-myc antibodies, respectively. Nephrin co-precipitated Neph3-myc (lane 2) and nephrin was detected in Neph3-myc precipitate (lane 4). Control rabbit (lane 3) and mouse (lane 5) IgG precipitations were negative. Nephrin and Neph3 expressing cell lysate (50 $\mu \mathrm{g})$ served as control (lane 1). (B) The extracellular domain of Neph3 pulled down nephrin and podocin (lane 2) from rat glomerular lysate whereas vector control did not (lane 3). Lane 1,50 $\mu \mathrm{g}$ of rat glomerular lysate. (C) The constructs encoding the intracellular fusion proteins of Neph1, nephrin, Neph3 and PKD1 were co-transfected with full-length Neph3-myc into 293T cells. The lysates were incubated with protein G-sepharose to precipitate the intracellular fusion proteins and immunoblotted with anti-myc to detect full-length Neph3-myc. Neph3-myc binds to intracellular domains of Neph1 (lane 2), nephrin (lane 3) and Neph3 (lane 4) whereas the control precipitation containing the intracellular domain of PKD1 (lane 1) was negative. IP, immunoprecipitation; IB, immunoblot; PD, pull down.

Figure 2. Characterization of L-cells stably expressing nephrin, Neph1-HA, Neph3-EGFP and EGFP by immunoblotting and immunofluorescence. (A) Immunoblot analysis of L-cells stably expressing nephrin either alone (lane 4) or together with Neph3-EGFP (lane 5) showed that nephrin has a similar molecular weight in L-cells as endogenous nephrin in rat glomerular lysates (lane 1). EGFPtagged Neph3 also migrated at the expected molecular weight in L-cells stably expressing Neph3-EGFP alone (lane 3) or in combination with nephrin (lane 5). Control cells expressed EGFP (lane 2). (B) Neph1-HA stably expressed in L-cells either alone (lane 2) or together with nephrin (lane 4) migrated at its expected molecular weight. Nephrin expression was confirmed in L-nephrin (lane 3) and Lnephrin-Neph1-HA (lane 4) cells. In A-B, L-cells were infected with retroviruses carrying nephrin, Neph3-EGFP and Neph1-HA and selected with G418, puromycin or hygromycin to obtain stable cell lines. Rat glomerular lysate and L-cell lysates (75 $\mu \mathrm{g})$ were separated by SDS-PAGE followed by immunoblotting with anti-nephrin, anti-GFP and anti-HA antibodies. Actin was used as a loading control. (C) Immunofluoresence staining showed that nephrin localizes mainly in free membrane edges in L-nephrin cells (arrows). (D) The localization of nephrin on the plasma membrane of L-nephrin cells was confirmed by surface staining. (E) Neph3-EGFP and (F) Neph1-HA localized in cell-cell contacts when stably expressed in L-cells (arrows) and (G) EGFP was found in cytosol. In C-G, cells were fixed with acetone or alternatively with PFA (Neph1-HA and nephrin surface stainings) and immunolabeled with anti-nephrin (L-nephrin) or anti-HA (L-Neph1-HA) antibodies followed by examination by immunofluorescence. Scale bar $20 \mu \mathrm{m}$. IB, Immunoblot.

Figure 3. Homophilic and heterophilic adhesion activities of nephrin, Neph1 and Neph3. (A) L-nephrin and L-nephrin-Neph1-HA cells did not show significant aggregation compared to control cells (L-Mock) in hanging drop assay. L-Neph1-HA cells aggregated 2.1-times more than L-Mock cells $(p<0.001)$. Combination of Lnephrin and L-Neph1-HA cells in hanging drop assay showed 3-times increased aggregation index in comparison with L-Mock cells ( $\mathrm{p}<0.001)$. (B) L-nephrin-Neph3EGFP cells showed a minor 1.4-fold increase in aggregation index compared to L- 
EGFP cells $(\mathrm{p}<0.01)$ in hanging drop assay. L-Neph3-EGFP cells aggregated 2.3times more $(\mathrm{p}<0.001)$ and combination of L-nephrin and L-Neph3-EGFP cells 3times more $(\mathrm{p}<0.001)$ than L-EGFP cells in hanging drop assay. In A-B, the bars represent an average $( \pm \mathrm{SD})$ of three independent experiments with three replicates in each experiment $(\mathrm{n}=9)$. Statistical analysis was done by two-tailed paired $t$-test; $* *$ $\mathrm{p}<0.01$, *** $\mathrm{p}<0.001$. (C) Immunofluoresence stainings of L-nephrin and L-Neph3EGFP cell aggregates after $24 \mathrm{~h}$ in hanging drop culture. Merged image shows colocalization of nephrin and Neph3-EGFP in cell-cell contacts (arrow) in the cell aggregate. Scale bar $20 \mu \mathrm{m}$.

Figure 4. Heterophilic adhesion between nephrin and Neph1 or Neph3 results in decreased total tyrosine phosphorylation of nephrin. (A) Quantitative immunoblot analysis showed that total tyrosine phosphorylation of rat nephrin stayed at similar level in L-nephrin, L-nephrin-Neph3-EGFP and L-nephrin-Neph1-HA cells in hanging drop cultures. However, combination of L-nephrin and L-Neph3-EGFP cells allowing trans-interaction of the proteins showed $51 \%$ decrease $(\mathrm{p}<0.01)$ and combination of L-nephrin and L-Neph1-HA cells showed $48 \%$ decrease $(p<0.05)$ in total tyrosine phosphorylation of nephrin compared to L-nephrin cells in hanging drop cultures. Bars represent an average $( \pm S D)$ of four independent experiments. Nephrin was immunoprecipitated from $24 \mathrm{~h}$ hanging drop culture lysates and immunoblotted with anti-nephrin and anti-phospho-tyrosine antibodies. Relative tyrosine phosphorylation of nephrin was quantified by normalizing the amount of phosphotyrosine to total amount of nephrin in nephrin immunoprecipitates. Statistical analysis was done by two-tailed paired $t$-test; $* \mathrm{p}<0.05$, ** $\mathrm{p}<0.01$. Representative immunoblot is shown on the right. (B) The intracellular domain of rat nephrin contains seven putative tyrosine phosphorylation sites. Tyr1204 and Tyr1228 are flanked by conserved amino acid residues which upon phosphorylation of the tyrosine residues can serve as binding sites for $\mathrm{SH} 2$ domain containing proteins. (C-D) Immunoblotting of hanging drop culture lysates $(100 \mu \mathrm{g})$ from L-cells with phosphospecific nephrin antibodies pY1193 (C) and pY1217 (D), which recognize phosphorylated Tyr1204 and Tyr1228 sites of rat nephrin, respectively, showed a positive signal in nephrin expressing cells and a negative signal in L-Mock and LEGFP cells, confirming the specificity of the antibodies. Actin was used as a loading control. (E-F) Quantitative immunoblot analysis showed no significant alterations in phosphorylation of rat nephrin at Tyr1204 (E) or Tyr1228 (F) between L-nephrin, combination of L-nephrin and L-Neph3-EGFP and combination of L-nephrin and LNeph1-HA cells in hanging drop cultures. The bars represent an average $( \pm S D)$ of three independent experiments with three replicas in each experiment $(n=9)$. Representative immunoblots are shown on the right. In E-F, cell lysates from $24 \mathrm{~h}$ hanging drop cultures were immunoblotted with nephrin and nephrin phosphospecific antibodies pY1193 and pY1217. The amount of tyrosine-phosphorylated nephrin was normalized to total amount of nephrin. IP, immunoprecipitation; IB, immunoblot.

Figure 5. Neph3 is up-regulated in nephrin deficient mouse kidneys. (A) Quantitative immunoblot analysis showed 4-fold increase $(p<0.01)$ in Neph3 expression in nephrin ${ }^{\text {trap/trap }}$ mouse kidneys (-/-) compared to wild-type controls $(+/+)$. Neph1 expression was also increased 2-fold in neprin ${ }^{\text {trap/trap }}$ kidneys, but did not reach statistical significance $(p=0.14)$. Level of podocin showed no alterations between nephrin $^{\text {trap/trap }}$ and wild-type kidneys and hardly any nephrin was detected in 
nephrin $^{\text {trap/trap }}$ kidneys as expected. The immunoblot analysis was repeated with similar results $(n=5$ per genotype). Tubulin was used for normalization and statistical analysis was done by two-tailed homoscedastic $t$-test; ** $\mathrm{p}<0.01$ and $* * * \mathrm{p}<0.001$. (B) Representative immunoblot of quantifications in (A). (C) Immunofluorescence staining showed that Neph3 localizes in the podocytes lining capillary loops in both wildtype $\left(\right.$ nephrin $^{+/+}$) and nephrin deficient kidneys (nephrin ${ }^{\text {trap/trap }}$ ). Nephrin localized in podocytes in wildtype kidneys and was not detected in nephrin deficient kidneys. Scale bar $50 \mu \mathrm{m}$. IB, immunoblot. 


\section{Figure 1.}

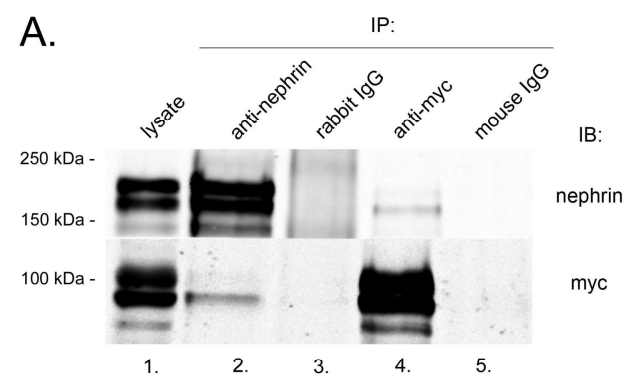

B.

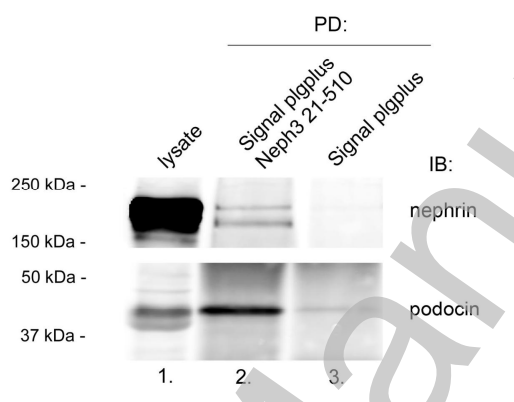

C.

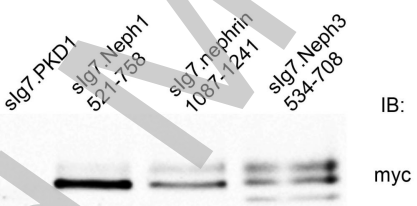

2.

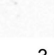




\section{Figure 2.}

A.

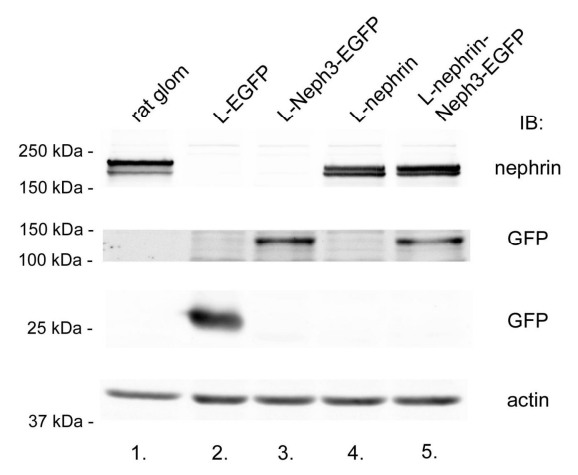

B.

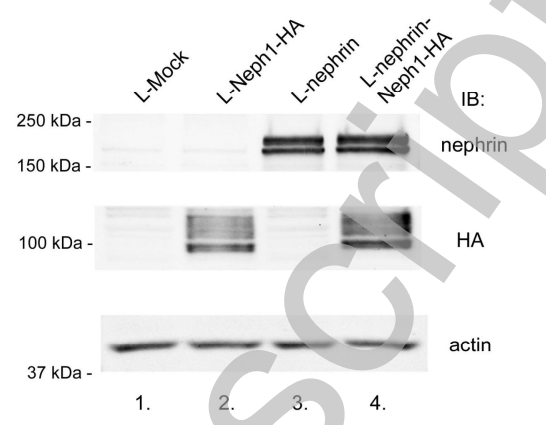

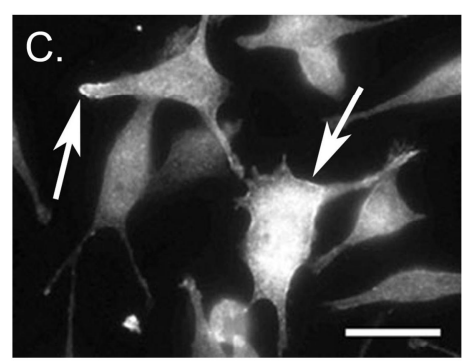
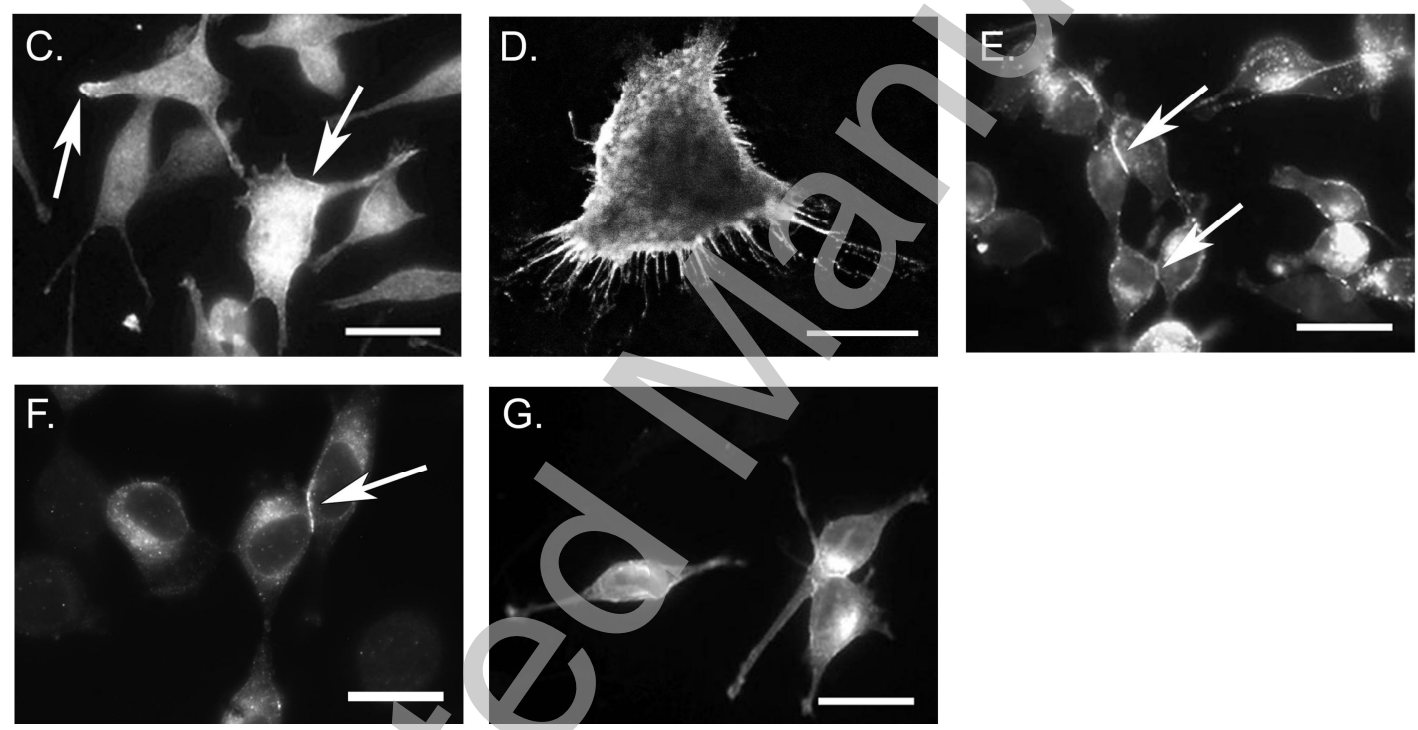

Licenced copy. Copying is not permitted, except with prior permission and as allowed by law. (c) 2011 The Authors Journal compilation (c) 2011 Portland Press Limited 


\section{Figure 3.}

A.

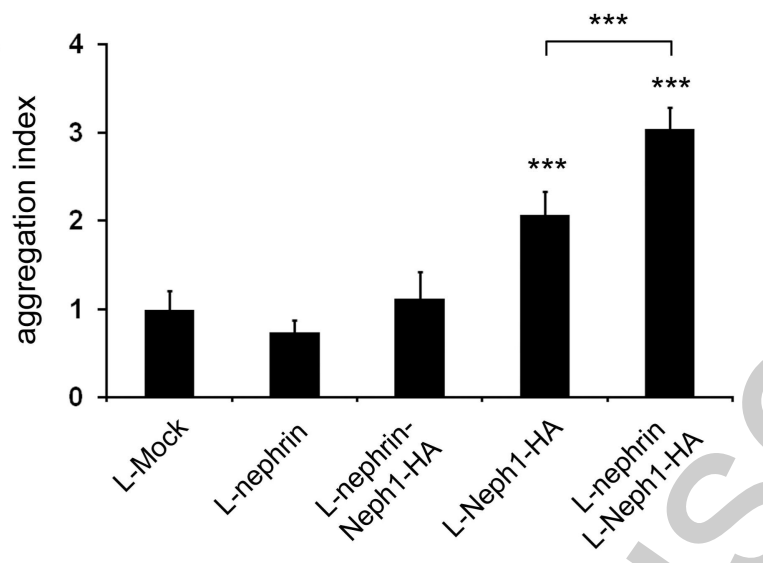

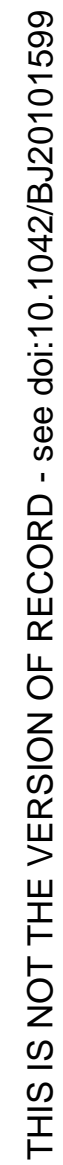

B.

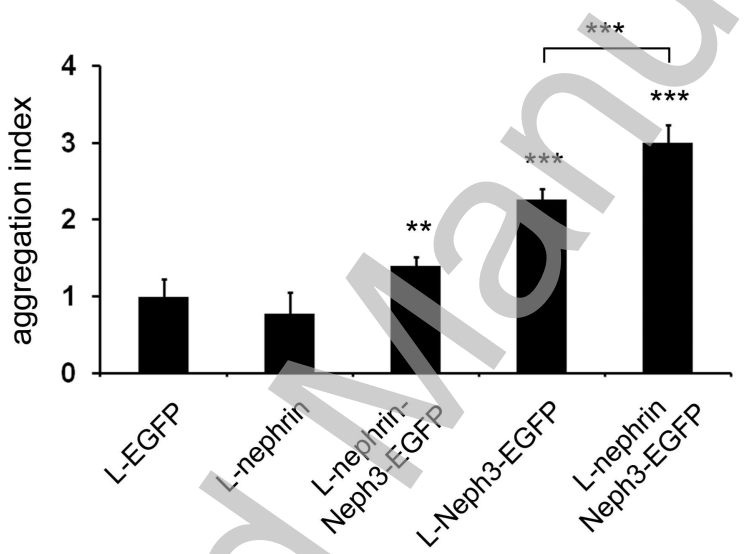

C.

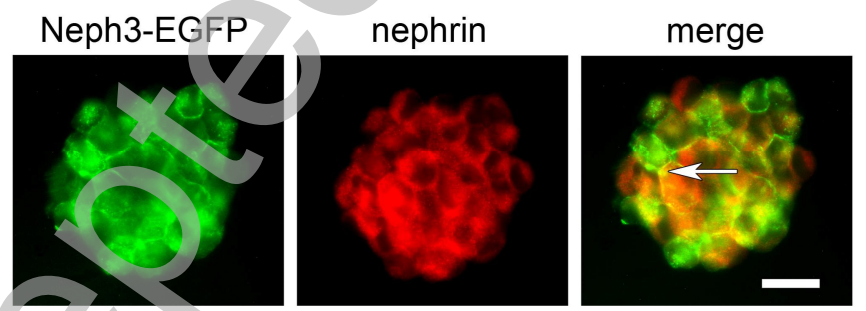




\section{Figure 4 .}

A. Relative total rat nephrin tyrosine phosphorylation (\%)
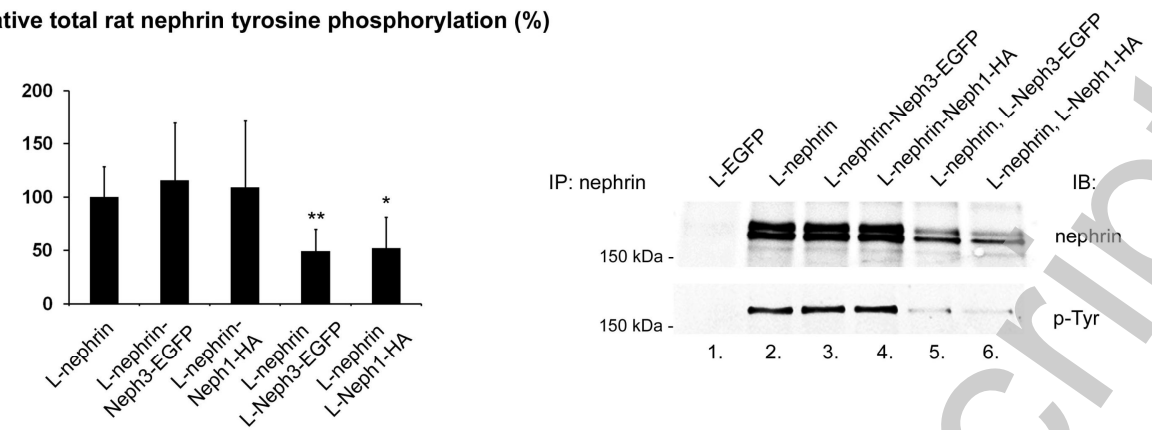

B.

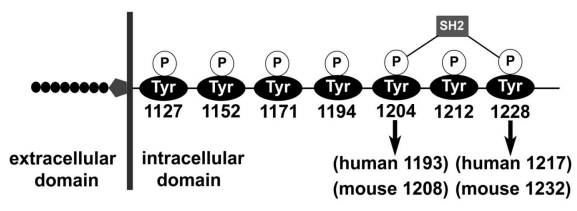

C.

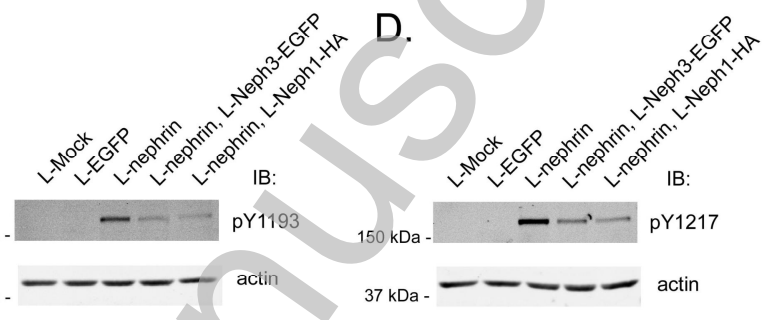

E. Relative tyrosine phosphorylation of rat nephrin at Tyr1204 (\%)
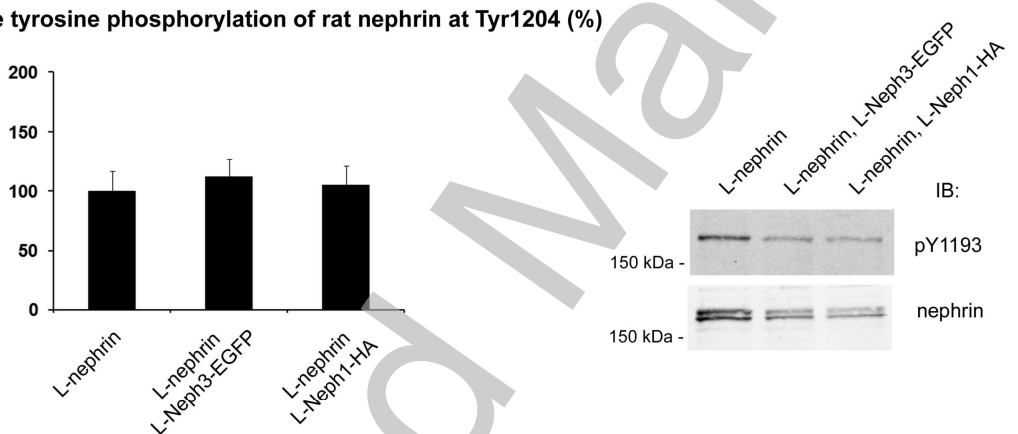

F Relative tyrosine phosphorylation of rat nephrin at Tyr1228 (\%)

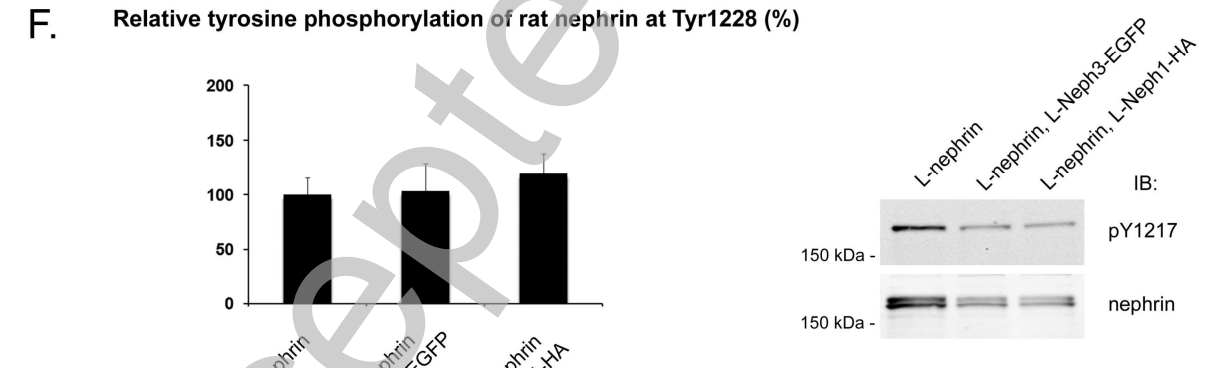
$150 \mathrm{kDa}$ 


\section{Figure 5.}

A.
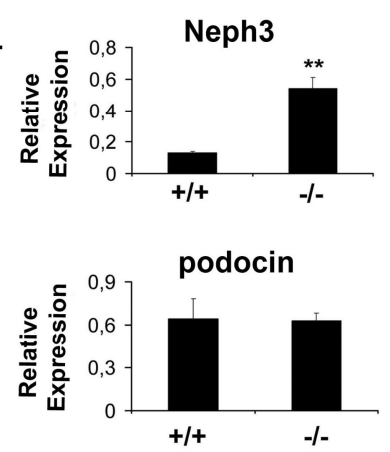
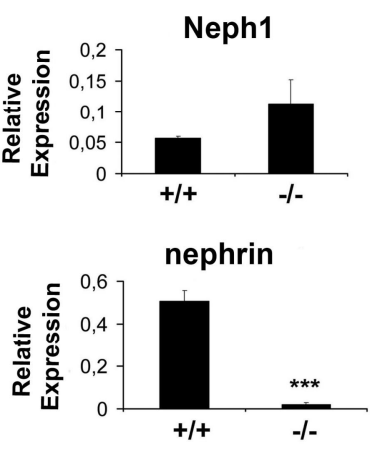

B.

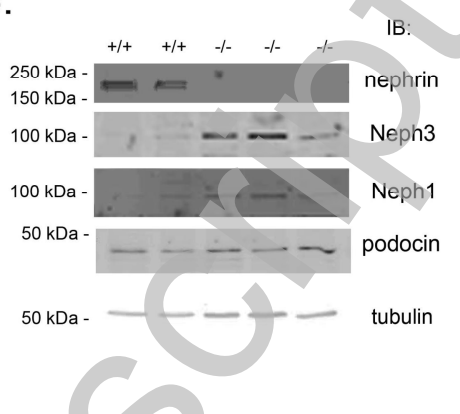

C.

nephrin $^{\text {trap/trap }}$

nephrin $^{+/+}$

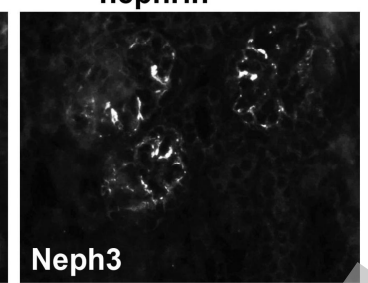

nephrin trap/trap

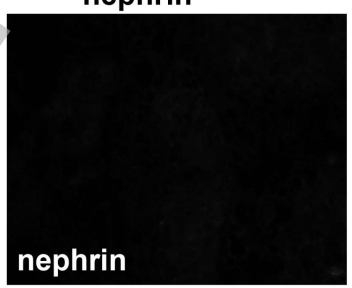

